\title{
Fatores associados ao padrão de consumo alimentar da população brasileira urbana
}

\author{
Factors associated with dietary patterns \\ in the urban Brazilian population
}

Rosel y Sichieri 1

Joelma Ferreira Gomes Castro 1

Aníbal Sanchez Moura 2

\footnotetext{
1 Instituto de Medicina Social, Universidade do Estado do Rio de Janeiro. Rua São Francisco Xavier 524 Bloco E, Rio de Janeiro, RJ 20550-030, Brasil. sichieri@uerj.br

2 Laboratório de Fisiologia e Desenvolvimento da Nutrição, Instituto de Biologia, Universidade do Estado do Rio de Janeiro. Av. 28 de Setembro $s / n$, Hospital Universitário Pedro Ernesto, Rio de Janeiro, RJ 20550-030, Brasil.
}

\begin{abstract}
This study evaluated factors associated with di etary patterns in the Brazilian population based on the Living Standards Survey conducted in the Northeast and Southeast regi ons of the country. Multi-stage probability sampling was employed to select the households, and for the present analysi s 5,121 adults aged 20 to 50 years were included. Pregnant women and individuals reporting chronic health conditions were excluded. Through principal component analysis, two major family dietary patterns were identified: a mixed pattern, in which all groups and foods have approximately the same factor loading, and a second pattern based main ly on rice and beans, which was called a traditional diet. Weight and height were measured in the households, and food intake was based on a 21-item semi-quantitative family questionnaire. The Northeast (as compared to the Southeast) was negatively associated with the mixed pattern. Body mass index was positively associated with the mixed pattern, whereas lei sure physical activity and Black skin color were negatively associated with the mixed pattern. Schooling and income levels explained most of the di etary variance, but after adjusting for education and income, region of residence remained significantly associated and was the third most important explanatory variable.

Key words Nutrition Surveys; Body Mass Index; Feeding Patterns
\end{abstract}

Resumo Avaliou-se os fatores que explicariam diferentes padrões de consumo alimentar da população urbana brasileira, com base na Pesquisa sobre Padrões de Vida, realizada no Nordeste e no Sudeste em 1996-1997. Foram incluídos os dados de consumo familiar (últimos 14 dias a partir de uma listagem com 28 alimentos) de 5.121 adultos na faixa etária de 20 a 50 anos, após exclusão das gestantes, das lactantes e das pessoas que referiram problema de saúde crônico. Os padrões de consumo alimentar foram definidos pela análise de componentes princi pais, ondeo valor de carga de uma determinada variável (alimento) é proporcional à sua contribuição para o fator (padrão de consumo) em questão, com a carga negativa indicando que a variável está inversamente associada com o fator, enquanto a posi tiva indica uma associação direta. A Região Nordeste comparada à Regi ão Sudeste associ ou-se negati vamente a um padrão misto, quando todos os al imentos foram igual mente importantes. O índice de massa corporal associou-se positivamente ao padrão misto, e praticar atividade física e ser negro associou-se negativamente a este padrão. Escolaridade e renda foram as variáveis que mais expli caram o consumo alimentar, mas mesmo ajustando por elas, a região de residência foi o tercei ro mai or componente explicativo. Palavras-chave Inquéritos Nutricionais; Índice de Massa Corporal; Padrões Alimentares 


\section{Introdução}

A maioria dos trabal hos de avaliação do consumo alimentar de populações baseia-se no cômputo dos macronutrientes, respectivo consumo calórico e no consumo daqueles micronutrientes mais freqüentemente associados ou às deficiências ou às doenças crônicas não transmissíveis da referida população (Hankinson et al., 2001; M argetts \& Nelson, 1991; Willett, 1998). Mais recentemente, os alimentos, ao invés de nutrientes, têm sido valorizados e, já em 1998, a Organização Mundial da Saúde - OMS - (WHO, 1998) sugeria que as recomendações alimentares para populações deveriam basear-se em alimentos ao invés de nutrientes. Para o Brasil, há pelo menos duas publicações que enfatizam propostas de recomendações de consumo centradas em alimentos (Instituto Danone, 1998; Sichieri et al., 2000b) e, no âmbito da pesquisa em Nutrição, essa tendência deu origem a estudos de avaliação de padrões de consumo al imentar (Go et al., 2001; Heber \& Bowerman, 2001; Simopoulos, 2001).

Para a população do M unicípio do Rio de Janeiro, Sichieri (2002) avaliou padrões de consumo alimentar, obtidos pela metodologia de componentes principais, e mostrou que um padrão de consumo al imentar tradicional, baseado em arroz e feijão, foi protetor para a presença de sobrepeso e obesidade. Vale salientar que os dados da referida pesquisa, de corte transversal para o Município do Rio de Janeiro, quando avaliados em relação aos macronutrientes, não mostraram associação com obesidade (Sichieri et al., 2000a), indicando que padrões de consumo alimentar sejam talvez as formas mais abrangentes de explorar o consumo.

Teoricamente, os padrões de consumo permitem o entendimento de construções sociais, ecologicamente determinadas, e orientadas ao provimento de condições de reprodução de populações. Ou seja, diferentes padrões de consumo refletem situações nas quais as populações associando-se de forma multideterminada a cadeias alimentares tendem a desenvolver um maior equilíbrio (auto-organização $=$ menor morbidade) ou, contrariamente, são induzidas a situações de menor equilíbrio e de auto-desorganização = alta morbidade (Sneppen et al., 1995). Portanto, é da natureza dos padrões de consumo alimentar a sua maior ou menor capacidade de geração (ou não) da saúde. Dessa forma, o padrão de consumo de alimentos, mais que a ausência específica de nutrientes na dieta, expressa situações reais de disponibilidade de alimentos e de condições diferenciadas de inserção ou não das populações nos diferentes cenários sociais.
Explorar os padrões de consumo alimentar, sua associação com fatores sócio-econômicos, demográficos, atividade física de lazer e com o sobrepeso nas duas maiores regiões urbanas do Brasil, foi o objetivo do presente trabalho. Mais especificamente, buscou-se explorar os fatores que explicariam diferentes padrões de consumo da população urbana brasileira.

\section{Metodologia}

A Pesquisa sobrePadrões deVida (PPV), da Fundação Instituto Brasileiro de Geografia e Estatística (IBGE, 1998), foi realizada entre março de 1996 e março de 1997, nas regiões Nordeste e Sudeste do Brasil. A descrição sobre amostragem encontra-se na documentação do IGBE (1998), em Castro (2001) e em Monteiro et al. (2001). Em resumo, foram avaliados somente o Nordeste e o Sudeste pois estas áreas equivalem a dois terços da população brasileira e apresentam os extremos do desenvolvimento desigual do Brasil. Nessas duas regiões, foram considerados oito estratos geográficos na área urbana e, em cada um deles, três níveis de renda. Na presente análise foram incluídos adultos na faixa etária de 20 a 50 anos, com exceção de gestantes (205), lactantes (77) e pessoas que apresentavam problema de saúde crônico (1.224). Excluíram-se adultos com mais de 50 anos, para evitar que mulheres em pós-menopausa, que teriam maior propensão a modificar o consumo alimentar, compusessem a amostra (5.121 adultos).

O questionário utilizado no inquérito foi o da pesquisa do Banco Mundial, Living Standard Measurements (LSMS). A listagem dos alimentos incluídos baseou-se nas pesquisas de orçamento familiar do IBGE, e o consumo alimentar avaliado foi medido pelo consumo de alimentos ou grupos de alimentos, referente aos últimos 14 dias pela unidade familiar. U ma lista com 28 alimentos e seus equivalentes em quantidades compunham o questionário. Dos 28 alimentos, foram excluídos da análise os itens café, chá e mate, que compunham um item do questionário, e gorduras e óleos vegetais, que também foram agrupados no questionário. Na definição do padrão alimentar, assumiu-se que gorduras e óleos poderiam significar padrões muito diferentes, o mesmo ocorrendo para café e chá, e portanto estes itens foram excluídos das análises. Bebidas também foram excluídas, por não ser possível identificar o tipo da bebida, se alcoólica ou não; e leite, por ser um alimento marcador do consumo alimentar infantil; temperos, molhos e condimentos; doces e balas, pois estes foram referidos em mais de 16 uni- 
dades de medida diferentes. Os alimentos e grupos de alimentos incluídos na presente análise encontram-se na Tabela 1.

Os padrões de consumo al imentar foram definidos a partir da análise de componentes principais utilizando o programa Statistical Analysis System (SAS), versão 6.12. Neste tipo de análise a carga negativa (-) indica que a variável está inversamente associada com o fator, enquanto a carga positiva (+) indica uma associação direta. O valor de carga de uma determinada variável é proporcional à sua contribuição para o fator em questão (Kleinbaum et al., 1988). Na presente análise foram retidos os padrões com eingenvalues $>1,5$. Eingenvalues são influenciados pelo número total de componentes da análise. A somatória dos eingenvalues do total dos padrões é igual ao número total de componentes, sendo 21 no presente estudo. Foram selecionados dois padrões de consumo familiar (Tabela 1) e os componentes principais categorizados em quartis. A partir dos quartis criou-se uma variável composta (padrão geral de consumo) pela soma dos dois quartis. Essa variável identifica desde os mais aderentes à dieta tradicional aos mais aderentes à dieta mista. Categorizamos de 1 a 4 para os valores crescentes do componente 1 (misto) e de 4 a 1 para os crescentes do componente 2 (tradicional). Dessa forma, o indivíduo com padrão geral de consumo familiar (variável composta) igual a 2, estaria no primeiro quartil do componente misto e no quarto do componente tradicional. 0 valor 8 significa a mais alta aderência possível ao padrão misto (Tabela 2).

Peso e altura foram medidos no domicílio por equipe previamente treinada. Somente $9,6 \%$ dos adultos não foram medidos. Para análise da associação entre padrão de consumo e sobrepeso, utilizou-se o Índice de Massa Corporal (IMC $=$ peso $\mathrm{em} \mathrm{kg} /(\text { altura })^{2} \mathrm{em} \mathrm{m}$ ) como variável contínua. Renda per capita em quintis inclui a renda da família dividida pelo número de moradores do domicílio. Escolaridade foi definida em seis grupos de anos de estudo. Cor foi definida pelo entrevistador como branca, parda, negra e amarela. Para análise agrupamos pardos e amarelos. Estado civil foi agrupado em solteiro, casado ou vivendo em companhia de cônjuge e uma categoria que incluiu os divorciados, desquitados e os viúvos. Atividade física de lazer, categorizada em sim ou não, foi avaliada na entrevista a partir da questão: pratica exercício físico ou esporte todas as semanas.
Tabela 1

Padrões de consumo alimentar, identificados segundo análise de componentes principais, das regiões urbanas do Nordeste e Sudeste brasileiro, 1997.

\begin{tabular}{lcc}
\hline $\begin{array}{l}\text { Alimentos e grupos } \\
\text { de alimentos }\end{array}$ & Padrão misto & Padrão tradicional \\
\hline Arroz, milho, outros cereais & 0,17 & 0,49 \\
Feijão, lentilha, ervilha, etc. & 0,20 & 0,50 \\
Tubérculos & 0,24 & $-0,11$ \\
Legumes & 0,30 & $-0,14$ \\
Verduras & 0,26 & $-0,14$ \\
Frutas, exceto em conserva & 0,36 & $-0,23$ \\
Farinha de mandioca & 0,15 & 0,26 \\
Pães & 0,14 & 0,00 \\
Bolos & 0,24 & $-0,26$ \\
Macarrão & 0,13 & 0,09 \\
Biscoitos & 0,24 & $-0,19$ \\
Açúcar & 0,25 & 0,30 \\
Sal & 0,02 & 0,04 \\
Derivados de leite & 0,28 & $-0,29$ \\
Peixes e crustáceos & 0,22 & $-0,02$ \\
Frango & 0,29 & 0,06 \\
Carne bovina & 0,28 & 0,12 \\
Carne suína & 0,11 & 0,09 \\
Outras carnes & 0,03 & $-0,06$ \\
Ovos & 0,17 & 0,11 \\
Enlatados prontos para consumo & 0,08 & $-0,08$ \\
Eingenvalue & 3,31 & 1,73 \\
\hline
\end{tabular}

Fonte: Pesquisa sobre Padrões de Vida (IBGE, 1998).

Tabela 2

Pontos de corte para os quartis dos componentes principais e categorização do padrão geral de consumo*, 1997.

\begin{tabular}{|c|c|c|c|c|}
\hline & \multicolumn{4}{|c|}{ Quartis } \\
\hline & 1 & 2 & 3 & 4 \\
\hline \multicolumn{5}{|l|}{ Padrão misto } \\
\hline Categorização & 1 & 2 & 3 & 4 \\
\hline Pontos de corte & $-0,66$ & $-0,17$ & 0,43 & \\
\hline \multicolumn{5}{|l|}{ Padrão tradicional } \\
\hline Categorização & 4 & 3 & 2 & 1 \\
\hline Pontos de corte & $-0,55$ & $-0,05$ & 0,50 & \\
\hline Padrão geral de consumo & \multicolumn{4}{|c|}{2 a 8} \\
\hline
\end{tabular}

* Soma das categorias dos dois componentes principais.

Fonte: Pesquisa sobre Padrões de Vida (IBGE, 1998). 


\section{Resultados}

$\mathrm{Na}$ Tabela 3, apresentamos as características da população adulta, excluídas as gestantes, lactantes e pessoas com problema de saú de crônico. Os valores médios de renda, IMC e idade foram maiores no Sudeste que apresentou, também, os maiores percentuais de brancos e a mai or escolaridade. O Sudeste apresentou, ainda, menor percentual de solteiros e famílias de menor tamanho.

Dois padrões de consumo alimentar foram selecionados: um padrão misto, no qual está presente o consumo de quase todos os alimentos e um tradicional, que apresenta características mais próximas da alimentação tradicional do brasileiro, destacando-se arroz, feijão, farinha e açúcar. Os eingenvalues desses dois padrões foram 3,31 e 1,73, respectivamente, e o terceiro padrão, que não foi considerado, apresentou eingenvalue de 1,10 (Tabela 1 ).

O padrão geral de consumo que combina os dois componentes principais (Tabela 2), associou-se a todas as variáveis estudadas (Tabela 4). A Região Nordeste comparada à Região Sudeste associou-se negativamente a um padrão com maior componente misto. O IMC associou-se positivamente ao padrão misto. Praticar atividade física e ter cor de pele mais escura associou-se a um menor consumo de dieta mista, ou seja, a maior aderência a um padrão tradicional. Das variáveis analisadas, escolaridade e renda foram as que mais explicaram o consumo alimentar, mas mesmo ajustando por elas, a região de residência foi o terceiro maior componente explicativo. As demais variáveis mostraram associação positiva com o padrão misto.

Tabela 3

Características da população de estudo, segundo regiões urbanas do Nordeste e Sudeste brasileiro, 1997

\begin{tabular}{|c|c|c|c|c|c|}
\hline & \multicolumn{2}{|c|}{ Nordeste } & \multicolumn{2}{|c|}{ Sudeste } & \multirow{2}{*}{$\begin{array}{l}\text { Valor de } p \text { do } \chi^{2} \\
\text { ou do teste } t\end{array}$} \\
\hline & Média & Desvio-padrão & Média & Desvio-padrão & \\
\hline Índice de massa corporal & 24,03 & 4,19 & 24,50 & & 0,0002 \\
\hline Renda per capita & 396 & 734 & 653 & 1.009 & $<0,0001$ \\
\hline Idade & 32,82 & 8,08 & 34,00 & 8,12 & $<0,0001$ \\
\hline \multirow[t]{2}{*}{ Tamanho do domicílio } & 4,91 & 2,21 & 4,24 & 1,87 & $<0,0001$ \\
\hline & $n$ & $\%$ & $\mathrm{n}$ & $\%$ & \\
\hline Cor & & & & & $<0,0001$ \\
\hline Branca & 812 & 31,7 & 1.724 & 67,3 & \\
\hline Parda & 1.551 & 60,6 & 695 & 27,1 & \\
\hline Negra & 197 & 7,7 & 142 & 5,6 & \\
\hline Escolaridade (em anos) & & & & & $<0,0001$ \\
\hline$<1$ & 240 & 9,4 & 108 & 4,2 & \\
\hline $1-3$ & 304 & 11,9 & 219 & 8,6 & \\
\hline $4-7$ & 707 & 27,6 & 852 & 33,3 & \\
\hline $8-10$ & 464 & 18,1 & 489 & 19,1 & \\
\hline 11 & 579 & 22,6 & 491 & 19,2 & \\
\hline $12 \mathrm{ou}+$ & 266 & 10,4 & 402 & 15,7 & \\
\hline Estado civil & & & & & $<0,0001$ \\
\hline Solteiro & 1.326 & 51,8 & 1.018 & 39,8 & \\
\hline Casado & 1.142 & 44,6 & 1.425 & 55,6 & \\
\hline Outros & 92 & 3,6 & 118 & 4,6 & \\
\hline Atividade física & & & & & 0,86 \\
\hline Sim & 513 & 20,0 & 508 & 19,8 & \\
\hline Não & 2.047 & 80,0 & 2.053 & 80,2 & \\
\hline
\end{tabular}

Fonte: Pesquisa sobre Padrões de Vida (IBGE, 1998). 


\section{Discussão}

O presente estudo permitiu caracterizar dois padrões de consumo na população urbana do Nordeste e Sudeste brasileiro. Esses padrões definidos como tradicional e misto foram também observados em análise prévia desses mesmos dados, quando as duas regiões e as áreas urbanas e rurais foram avaliadas em separado (Castro, 2001). A referida análise constatou que a metodologia superestimava a avaliação de consumo das áreas rurais e portanto, para avaliar os fatores associados aos padrões restringimos a análise à população urbana.

Outros estudos têm se utilizado da metodologia de identificação de padrões de consumo. Inicialmente, escores de consumo ou um perfil qualitativo foram usados (Campbell et al., 1982; Liu et al., 1999; Randall et al., 1990; Slaterry et al., 1998). Mais recentemente, diversos estudos têm classificado as dietas com base na técnica de análise de componentes principais (Barker et al., 1990; Fung et al., 2001a; Nicklas et al., 1989). A característica deste tipo de estudo, que claramente identifica diferentes padrões, é de não permitir definir quem na população adere aos padrões identificados. Para estudar a associação entre os padrões de consumo identificados e outras variáveis de estilo de vida e do meio ambiente, criamos uma variável somatória dos dois padrões identificados. Dessa forma, um extremo desta categorização somatória indica alta aderência ao padrão misto e o outro ao padrão tradicional. Escores de componentes principais também foram utilizados por Fung et al. (2001b), que identificaram entre as enfermeiras americanas um padrão de dieta chamado de prudente e um de ocidental, com o padrão ocidental associando-se positivamente com as doenças cardiovasculares.

No estudo de Framingham, padrões de consumo alimentar foram definidos por análise de cluster com base em questionários de freqüência de consumo, tendo sido identificados cinco padrões. Dos cinco padrões nenhum atendia às recomendações de consumo americano (Millen et al., 1996). Na Alemanha, metodologia semelhante encontrou oito clusters em estudo de base populacional. Quatro deles caracterizavam-se como dieta de má qualidade e as variáveis que se associaram aos padrões alimentares foram horas de sono, sexo, idade e nível sócioeconômico (Hulshol et al., 1992). A qualidade da dieta e sua associação com estilo de vida foi também estudada em idosos do estudo de Framingham e do estudo europeu SENECA (Haveman-Nies, 2001). Escores da dieta e clusters permitiram uma boa caracterização de consumo
Tabela 4

Coeficiente de regressão linear e coeficiente de correlação geral e parcial $\left(R^{2}\right)$, para variação do padrão geral de consumo alimentar*, 1997.

\begin{tabular}{llll}
\hline & $\beta$ & Valor de $p$ & R2 parcial \\
\hline Regiões (Nordeste/Sudeste) & $-0,70$ & $<0,04$ & 0,06 \\
Índice de massa corporal & 0,02 & $<0,0001$ & 0,003 \\
Renda per capita & 0,0001 & $<0,0001$ & 0,07 \\
Idade & 0,01 & $<0,0001$ & 0,004 \\
Tamanho do domicílio & $-0,15$ & 0,01 & 0,04 \\
Cor (branca = 1; parda =2; & $-0,11$ & 0,0023 & 0,0003 \\
negra = 3) & & & \\
Escolaridade (6 grupos) & 0,38 & $<0,0001$ & 0,11 \\
Estado civil (casado/outros) & 0,02 & 0,6017 & 0,00003 \\
Atividade física (sim/não) & $-0,14$ & 0,0132 & 0,001 \\
R2 total & & & 0,26 \\
\hline
\end{tabular}

* Soma das categorias dos dois componentes principais.

Fonte: Pesquisa sobre Padrões de Vida (IBGE, 1998).

associado a estilos de vida nesses dois estudos. Assim sendo, abordagens mais amplas do consumo alimentar, comparadas as tradicionais análises de nutrientes, têm sido cada vez mais utilizadas nos estudos de associações com patologias, inclusive com o câncer (Gerber, 2001).

Quanto à validade dos padrões alimentares, Hu et al. (1999) definiram padrões de consumo a partir de questionário de freqüência de consumo alimentar e mostraram alta validade e reprodutibilidade. Embora não tenham sido realizados estudos para validar os padrões encontrados para o Brasil, estudo no Rio de Janeiro de 1996, utilizando um questionário de consumo de freqüência semiquantitativo, previamente validado e utilizando como unidade de análise o indivíduo, encontrou três padrões, sendo que dois deles: misto e tradicional foram similares aos encontrados no presente estudo (Sichieri, 2002). As limitações do presente estudo, além da não validação do questionário utilizado incluem seu caráter transversal, onde por exemplo, o IMC poderia determinar o consumo e não vice-versa como avaliado; sua agregação do consumo na família, sabe-se que parte importante do consumo dos adultos, principalmente do homem, ocorre fora da unidade familiar (Sichieri, 1998). Contudo, os dados da presente análise para o Brasil são consistentes com os achados para o Rio de Janeiro, e reafirmam a possibilidade de definição dos padrões de consumo alimentar através da análise de componentes principais. Ainda em relação ao estudo realizado no Município do Rio de Janeiro, mesmo tendo como base dados transversais, foi possível mostrar que a dieta tradicional brasileira, baseada 
em arroz e feijão, era protetora para sobrepeso e obesidade (Sichieri, 2002), achado também consistente com os do presente estudo.

A presente análise permitiu concluir que vários fatores interferem nos padrões de consumo, desde o tamanho do domicílio, passando por variáveis sócio-econômicas, até variáveis do indivíduo como estado civil e cor. Saliente-se que mesmo após ajuste pelas variáveis anteriormente mencionadas, a região de residência mostrou-se associada de forma importante com os padrões de consumo, indicando que muitos são os fatores responsáveis pela determinação de um dado padrão de consumo alimentar. Estes resultados sugerem, ainda, que dificilmente va- riáveis associadas exclusivamente ao indivíduo permitem entender a complexidade de um fenômeno como o padrão de consumo alimentar.

Em conclusão, a caracterização de dois padrões de consumo reforça a idéia de que a emergência de fatores determinantes de patologias não surge da inexistência de nutrientes, porém da forma combinada com que estes interagem. Por exemplo, no estudo de padrões de consumo realizado neste trabalho, foi possível identificar um padrão de consumo tradicional no Brasil que é em grande parte determinado pelas condições sócio-econômicas, e que independente delas, mostra-se como fator de proteção para sobrepeso.
Referências

BARKER, M. E.; MCCLEAN, S. I.; THOMPSON, K. A. \& REID, N. G., 1990. Dietary behaviours and sociocultural demographics in Northern Ireland. British Journal of Nutrition, 64:319-329.

CAM PBELL, C.; ROE, D. A. \& EICKWORT, K., 1982. Qualitative diet indexes: A descriptive or an assessment tool? Journal of the American Dietetic Association, 81:687-694.

CASTRO, J. F. G., 2001. Padrões de Consumo Alimentar eĺndice de Massa Corporal nas Regiões Nordeste e Sudeste do Brasil. Dissertação de Mestrado, Rio de Janeiro: Instituto de Medicina Social, Universidade do Estado do Rio de Janeiro.

FUNG, T. T.; RIMM, E. B.; SPIEGEMAM, D.; RIFAI, N.; TOFLER, G. H.; WILLETT, W. C. \& HU, F. B., 2001a. Association between dietary patterns and plasma biomarkers of obesity and cardiovascular disease risk. American Journal of Clinical Nutrition, 73:6167

FUNG, T. T.; WILLETT, W. C.; STAMPFER, M. J.; MANSON, J. E. \& HU, F. B., 2001b. Dietary patterns and the risk of coronary heart disease in women. Archives of Internal Medicine, 161:1857-1862.

GERBER, M., 2001. The comprehensive approach to diet: A critical review. Journal of Nutrition, 131 (Sup. 11):3051S-3055S.

GO, V. L.; WONG, D. A. \& BUTRUM, R., 2001. Diet, nutrition and cancer prevention: Where are we going from here? Journal of Nutrition, 131(Sup. 11): 3121S-3168S.

HAVEMAN-NIES, A.; TUCKER, K. L.; DE GROOT, L. C.; WILSON, P. W. \& van STAVEREN, W. A., 2001. Eval- uation of dietary quality in relationship to nutritional and lifestyle factors in elderly people of the US Framingham Heart Study and the European SENECA study. European Journal of Clinical Nutrition, 55:870-880.

HANKINSON, S. E.; COLDITZ, G. A.; MANSON, J. E. \& SPEIZER, F. E., 2001. Healthy Women, Healthy Lives: A Guide to Preventing Disease, From the Landmark Nurses' Health Study. New York: Simon $\&$ Shuster Source.

HEBER, D. \& BOWERM AN, S., 2001. Applying science to changing dietary patterns. Journal of Nutrition, 131(Sup. 11):3078S-3081S.

HU, F. B.; RIMM, E.; SMITH-WARNER, S. A.; FESKANICH, D.; STAMPFER, M. J.; ASCHERIO, A.; SAM PSON, L. \& WILLETT, W. C., 1999. Reproducibility and validity of dietary patterns assessed with a food-frequency questionnaire. American Journal of Clinical Nutrition, 69:243-249.

HULSHOL, K. F.; WEDEL, M.; LOWIK, M. R.; KOK, F. J.; KISTEM AKER, C.; HERMUS, R. J.; TEN HOOR, F. \& OCKHUIZEN, T., 1992. Clustering of dietary variables and other lifestyle factors (Dutch Nutritional Surveillance System). Journal of Epidemiology and Community Health, 46:417-424.

IBGE (Fundação Instituto Brasileiro de Geografia e Estatística), 1998. Pesquisa sobre Padrões de Vida PPV, 1996-1997. Rio de Janeiro: IBGE.

INSTITUTO DANONE, 1998. Alimentação Equilibrada para a População Brasileira. Florianópolis: Instituto Danone.

KLEINBAUM, D. G.; KUPPER, L. L. \& MULLER, K. L., 
1988. Applied Regression Analysis and Other Multivariate Methods. Boston: PWS-KENT Publishing Company.

LIU, S.; STAM PFER, M. J.; HU, F. B.; GIOVANNUCCI, E.; RIMM , E.; MANSON, J. E.; HENNEKENS, C. H. \& WILLETT, W. C., 1999. Whole-grain consumption and risk of coronary heart disease: Results from the Nurses' Heath Study. American Journal of Clinical Nutrition, 70:412-419.

MARGETTS, B. M. \& NELSON, M., 1991. Design Concepts in Nutritional Epidemiology. Oxford: Oxford University Press.

MILLEN, B. E.; QUATROM ONI, P. A.; GAGNON, D. R.; CUPPLES, L. A.; FRANZ, M. M. \& D'AGOSTINO, R. B., 1996. Dietary patterns of men and women suggest targets for health promotion: The Framingham Nutrition Studies. American Journal of Health Promotion, 11:42-53.

MONTEIRO, C. A.; CONDE, W. L. \& POPKIN, B. M., 2001. Independent effects of income and education on the risk of obesity in the Brazilian adult population. Journal of Nutrition, 131:881S-886S.

NICKLAS, T. A.; WEBBER, L. S.; THOMPSON, B. \& BERENSON, G. S., 1989. A multivariate model for assessing eating patterns and their relationship to cardiovascular risk factors: The Bogalusa Heart Study. American Journal of Clinical Nutrition, 49: 1320-1327.

RANDALL, E.; MARSHALL, J. R.; GRAHAM , S. \& BRASURE, J., 1990. Patterns in food use and their associations with nutrient intakes. American Journal of Clinical Nutrition, 52:739-745.
SNEPPEN, K.; BAK, P.; FLYVBJERG, H. \& JENSEN, H. M., 1995. Evolution as a self-organized critical phenomenon. Proceedings of the National Academy of Science of the United States of America, 92: 5209-5213.

SICHIERI, R., 1998. Epidemiologia da Obesidade. Rio de Janeiro: Eduerj.

SICHIERI, R., 2002. Dietary patterns and their associations with obesity in the Brazilian city of Rio de Janeiro. Obesity Research, 10:42-49.

SICHIERI, R.; SIQUEIRA, K. S. \& MOURA, A. S., 2000a. Obesity and abdominal fatness associated with undernutrition early in life in a survey in Rio de Janeiro. International Journal of Obesity and Related Metabolic Disorders, 24:614-618.

SICHIERI, R.; COUTINHO, D. C.; MONTEIRO, J. B. \& COUTINHO, W. F., 2000b. Recomendações de alimentação e nutrição saudável para a população brasileira. Arquivos Brasileiros de Endocrinologia e M etabologia, 44:227-232.

SLATERRY, M. L.; BOUCHER, K. M.; CAAN, B. J.; POTTER, J. D. \& MA, K., 1998. Eating patterns and risk of colon cancer. American Journal of Epidemiology, 148:4-16.

SIM OPOULOS, A. P., 2001. The Mediterranean Diets: What is so special about the diet of Greece? The scientific evidence. Journal of Nutrition, 13:3065S30735 .

WILLET, W., 1998. Nutritional Epidemiology. 2nd Ed. New York: Oxford University Press.

WHO (World Health Organization), 1998. Report of a Joint FAO/WHO Consultation. Preparation and Use of Food-Based Dietary Guidel ines. Geneva: WHO.

Recebido em 28 de dezembro de 2001 Versão final reapresentada em 6 de maio de 2002 Aprovado em 20 de junho de 2002 